\title{
Tuning of Color Chromaticity of Light Emission from ZnSe Films Grown on a GaAs Substrate by Atomic Layer Epitaxy
}

\author{
M. Skrobot ${ }^{a}$, M. Godlewski ${ }^{a, b}$, E. GuzIEWICZ ${ }^{b}$ \\ K. KOPAlKo ${ }^{b}$ AND M.R. Phillips ${ }^{c}$ \\ ${ }^{a}$ Dept. of Mathematics and Natural Sciences, College of Science
} Cardinal S. Wyszyński University, Dewajtis 5, 01-815 Warsaw, Poland

${ }^{b}$ Institute of Physics, Polish Academy of Sciences

al. Lotników 32/46, 02-668 Warsaw, Poland

${ }^{c}$ Microstructural Analysis Unit, UTS, Sydney, Australia

\begin{abstract}
Monocrystalline films of sphalerite-type ZnSe were grown on $\operatorname{GaAs}(100)$ substrates from elemental $\mathrm{Zn}$ and Se precursors by atomic layer epitaxy in a gas flow system. Due to color mixing of band edge and deep defect-related emissions these layers emit intensive white light. Depth profiling cathodoluminescence indicates that green and red emissions mostly come from disordered regions of the films, close to the ZnSe/GaAs interface. We tested a possibility of tuning of chromaticity coordinates and of color temperature of the emission. We found that the chromaticity parameters (color perception) can be tuned by either regulating the appropriate accelerating voltage of electrons or current density of primary electrons in cathodoluminescence investigations. These properties of ZnSe films make them suitable for some practical applications as white light sources.
\end{abstract}

PACS numbers: 42.79.--e, 78.66.--w, 81.05.--t

\section{Introduction}

Semiconductor-based light emitting devices are expected to replace conventional light sources, which are either of a low efficiency (incandescent lamps) or contain mercury pollution (metal-halide lamps and fluorescent lamps). There are several approaches to achieve white color emission from semiconductor sources. The most prospective one is hybrid white light emitting device (LED) in which blue emission from InGaN quantum well is mixed with yellow emission of phosphor deposited on top of LED [1, 2]. All commercialized semiconductor LEDs 
are based on III-V materials, whereas wide-gap II-VI compounds are used in commercialized thin film electroluminescence (TFEL) displays [3-5]. White color light from TFEL display is produced by mixing light from differently doped II-VI films and color filters are used for obtaining a required red/green/blue (RGB) proportion resulting in white light emission. An alternative idea exploits mixing of blue-green emission from $\mathrm{ZnCdSe/ZnSe} \mathrm{quantum} \mathrm{well} \mathrm{and} \mathrm{yellow} \mathrm{emission} \mathrm{from}$ a ZnSe substrate $[6,7]$. Our idea goes in a similar direction, but has a simplicity advantage over the former one. We propose to obtain white color emission from undoped ZnSe thin films deposited on GaAs substrate by atomic layer epitaxy (ALE) method. We have demonstrated $[8,9]$ that the excitation of ZnSe/GaAs layers results in effective white light emission. This emission is achieved by mixing so-called "edge" luminescence from monocrystalline ZnSe film and red and green light coming from defected $\mathrm{ZnSe} / \mathrm{GaAs}$ interface region. The supremacy of our approach is its remarkable simplicity both in the material structure (layer instead of QW) and the deposition method as well (ALE instead of MBE). Preliminary studies show [10] that chromaticity parameters (e.g. color temperature) of $\mathrm{ZnSe} / \mathrm{GaAs}$ monocrystalline films might be tuned by changing the ZnSe layer thickness. In the present study we demonstrate that adjusting of the accelerating voltage and current density assure the full range of color temperature from the same sample, e.g. $T_{\text {col }}$ ranges from $3000 \mathrm{~K}$ ("warm" indoor light) to $6000 \mathrm{~K}$ ("cold" light used for outdoor applications).

\section{Samples}

ZnSe samples were grown using an F-120 reactor of Microchemistry using nitrogen both as a transport agent and purging gas. The films were grown by

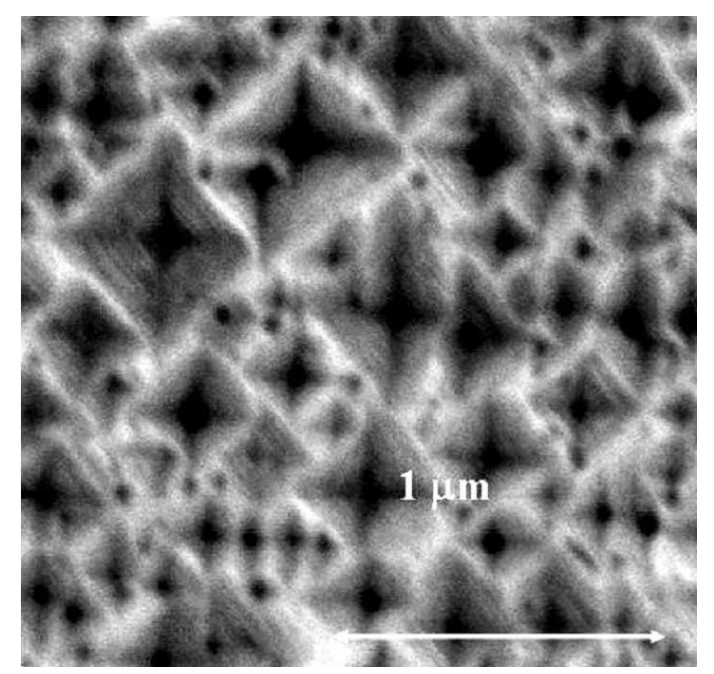

Fig. 1. SEM image of $2 \times 2$ region of $1.1 \mu$ m-thick ZnSe film grown on GaAs(100) substrate. The image was taken at $10 \mathrm{kV}$ and room temperature. 
synthesis reaction, e.g., using elemental zinc and selenium precursors. Uncoated, (100) oriented GaAs substrates were treated in $\mathrm{NH}_{4} \mathrm{OH}$ prior to the growth. Details of growth conditions can be found elsewhere [11]. In the present study we used films with thicknesses of $0.2-3.5 \mu \mathrm{m}$. Good crystalline quality of the films was confirmed by X-ray diffraction [9, 11], atomic force microscopy (AFM), and scanning electron microscopy (SEM). In Fig. 1 we show SEM image of $2 \times 2 \mu \mathrm{m}$ region of $1.1 \mu \mathrm{m}$-thick layer. The figure shows pyramidal pits of the same orientation characteristic of our ZnSe/GaAs(100) layers thicker than $0.2 \mu \mathrm{m}$.

\section{Optical characterization}

Optical properties of $\mathrm{ZnSe} / \mathrm{GaAs}$ films were characterized by photoluminescence (PL), micro-photoluminescence (micro-PL), and cathodoluminescence (CL). PL studies were performed for all the samples in temperature range 20-300 K using $488 \mathrm{~nm}$ or UV line of a coherent argon laser. Figure 2 demonstrates low

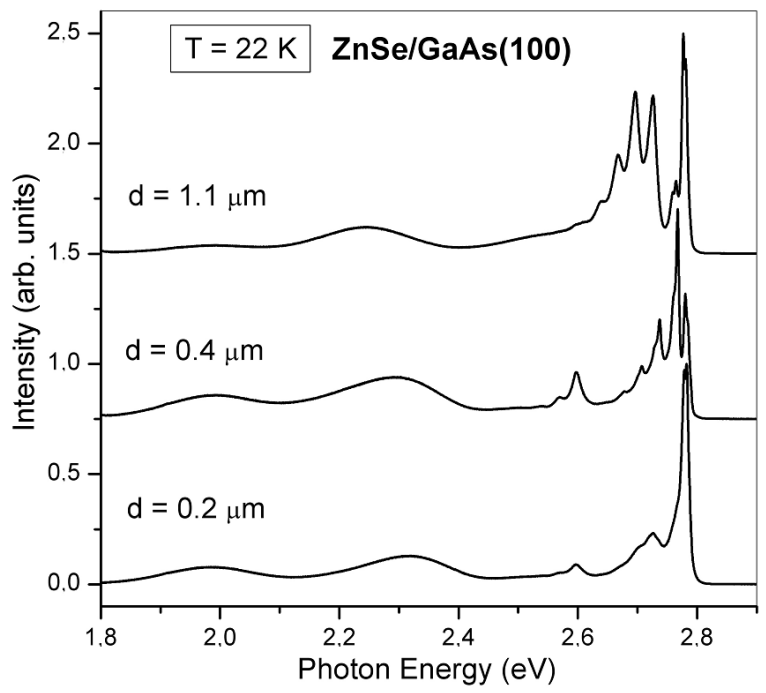

Fig. 2. Low temperature photoluminescence of $\mathrm{ZnSe} / \mathrm{GaAs}(100)$ films of different thicknesses $(d=0.2 \div 1.1 \mu \mathrm{m})$. All the spectra are normalized to a constant intensity.

temperature PL spectra of three samples with thicknesses between $0.2 \mu \mathrm{m}$ and $1.1 \mu \mathrm{m}$. A characteristic feature of all these spectra is blue "edge" luminescence of excitonic origin, which is observed in the photon energy region $2.7 \mathrm{eV}$ to $2.8 \mathrm{eV}$. The appearance of "edge" luminescence is a fingerprint of monocrystallinity of the ZnSe layer. The fine structure of "edge" PL changes with film thickness. Analysis of spectral position of the peaks lead to the conclusion that for layers thicker than 1.1 $\mu \mathrm{m}$ dominant PL lines are related to donor bound exciton (DBE) transitions with $\mathrm{Cl}$ or $\mathrm{Ga}$ acting as a donor [12]. For ZnSe/GaAs layers thinner than $0.5 \mu \mathrm{m}$ 
"edge" PL is dominated by acceptor bound exciton (ABE) transitions of unknown origin. This indicates changing of electrical properties of the films. Test electrical measurements confirm that thin $\mathrm{ZnSe} / \mathrm{GaAs}$ films are highly resistive, but became more $n$-type with increasing thickness.

In Fig. 2 there are also present two broad PL bands with maxima approximately at $2.0 \mathrm{eV}$ (red range of the spectrum) and $2.3 \mathrm{eV}$ (green range of the spectrum). Optically detected magnetic resonance (ODMR) investigations [13] proved that in this material $2.3 \mathrm{eV} \mathrm{PL}$ band is of donor-acceptor pair (DAP) origin related to $\mathrm{Cu}$ complexes acting as deep acceptors. The emission at $2.0 \mathrm{eV}$ was assigned to "self-activated" emission with zinc vacancy complex acting as a deep acceptor [13]. Two above-mentioned DAP emissions are hardly visible at low temperature, but start to be relatively more intensive at high temperature, where "edge" blue PL is thermally deactivated (Fig. 3).

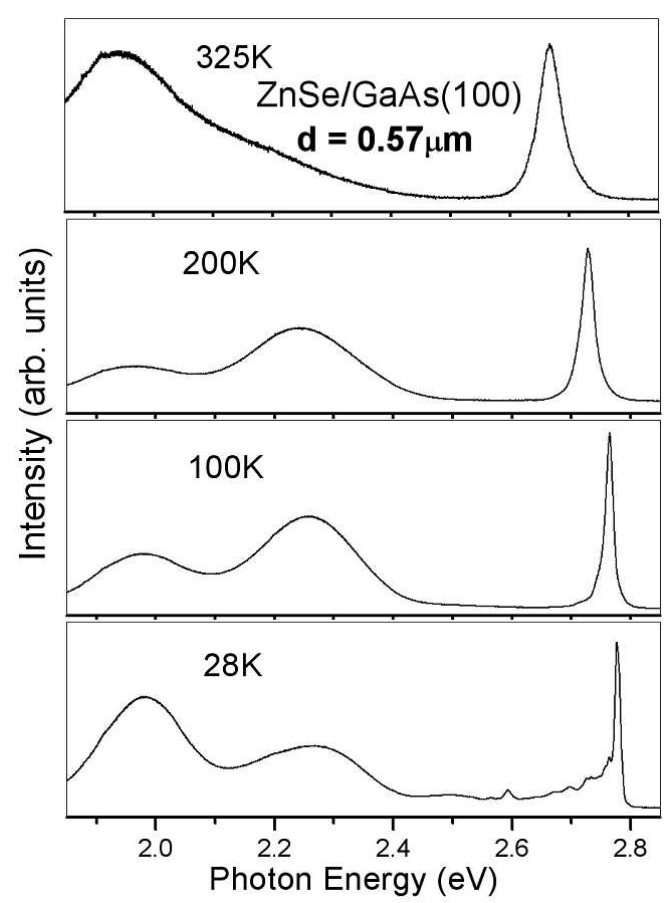

Fig. 3. Temperature dependence of the PL spectrum of $0.57 \mu \mathrm{m}$ thick ZnSe/GaAs film.

Micro-PL studies provide important information concerning origin of green and red DAP bands. As images obtained separately for green and red bands are not the same, we conclude that they are of different origin.

Mixing of red, green, and blue bands from $\mathrm{ZnSe} / \mathrm{GaAs}$ thin films results in a white light emission output. According to Commission Internationale d'Éclairage (CIE) each emission spectrum from a visible range $(380 \mathrm{~nm}$ to $760 \mathrm{~nm}$, which 
corresponds to $3.25 \mathrm{eV}$ to $1.62 \mathrm{eV}$ ) can be described by $x$ and $y$ coordinates at the so-called chromaticity diagram. Human eyes conceive light as a "white light", when the $(x, y)$ coordinates of its spectrum are located on the "black body radiator line" (black solid line in Fig. 6). Each point at the black body radiator line corresponds to a specific color temperature $\left(T_{\mathrm{col}}\right)$, and a light source with color temperature of $6000-7000 \mathrm{~K}$ is perceived as "cold white light" whereas a radiator with $T_{\text {col }}$ of $3000-3500 \mathrm{~K}$ as "warm white light". In practice, white color radiators with $T_{\text {col }}$ between $3000-6000 \mathrm{~K}$ are needed, depending on the range of application. For outdoor lighting we used sources characterized by $T_{\text {col }}=5000-6000 \mathrm{~K}$, whereas for indoor application sources with $T_{\text {col }}$ from the range of 3000-4500 K are applied.

Based on PL spectra we have made preliminary calculations of color temperature of light emitted in ZnSe/GaAs PL process (method of colorimetric calculations we described elsewhere [14]). Calculations show that ZnSe/GaAs layers of different thicknesses produce light output with the range of color temperature of 2400-4000 K, though $T_{\mathrm{col}}$ does not necessarily scale with the layer thickness. In the present study we investigated changing of light output from the same sample as a function of accelerating voltage and current density. The method of these studies described in the next paragraph is cathodolumincescence.

\section{Cathodoluminescence investigations}

Cathodoluminescence investigations have this advantage over PL ones that excitation conditions are achieved in the way analogous to that used in thin film electroluminescence (TFEL) devices, as in both cases the excitation medium is electron with energy of a few $\mathrm{kV}$.

CL investigations in a depth-profiling mode give information concerning in-depth distribution of red, green, and blue emissions from ZnSe/GaAs epilayers, as the average penetration range of primary electrons is a function of beam energy (Fig. 4). CL investigations with a varying accelerating voltage provide optical response from different parts of the film. For all the samples under investigation we observed the same characteristic of in-depth CL distribution. A smaller electron beam energy (about $6 \mathrm{kV}$ ) assures optimal conditions for a surface close excitation and excitonic blue luminescence dominates the spectra. At a larger beam energy (about $20 \mathrm{kV}$ ) emission is excited from the region close to the $\mathrm{ZnSe} / \mathrm{GaAs}$ interface and red and green emission bands dominate the spectra. As a result, we observe lower color temperature ("warm light") for higher accelerating voltage and higher color temperature ("cold light") for the lower one. Current density also influences the CL spectra, because for a high electron density some excitation channels saturate. In the case of $\mathrm{ZnSe} / \mathrm{GaAs}$ layers, we observe that red emission band saturates faster than "green" and "edge" emission channels (see Fig. 5) and we observe "green' shifting" in chromaticity diagram as seen in Fig. 6. Colorimetric parameters of $0.4 \mu \mathrm{m}$-thick and $0.57 \mu \mathrm{m}$-thick ZnSe films obtained for different accelerating voltages and current densities are gathered in Tables I and II. The $x$ 
TABLE I

Colorimetric parameters of $0.4 \mu \mathrm{m}$ thick $\mathrm{ZnSe} / \mathrm{GaAs}(100)$ thin film (open squares in Fig. 6). $I-$ current in $\mathrm{nA}$.

\begin{tabular}{c|c|c|c|c|c|c|c|c|c|c|c}
\hline \hline \multicolumn{10}{c}{ Accelerating voltage $10 \mathrm{kV}$} \\
\hline$I$ & 12.4 & 11.2 & 10.3 & 9.1 & 7.6 & 6.6 & 5.5 & 4.0 & 2.77 & 1.5 & 0.9 \\
\hline$x$ & 0.156 & 0.165 & 0.176 & 0.20 & 0.190 & 0.196 & 0.204 & 0.221 & 0.247 & 0.310 & 0.381 \\
$y$ & 0.088 & 0.093 & 0.104 & 0.128 & 0.123 & 0.127 & 0.135 & 0.150 & 0.171 & 0.244 & 0.263 \\
\hline \multicolumn{10}{c}{ Accelerating voltage $20 \mathrm{kV}$} \\
\hline$I$ & 11.0 & 9.9 & 8.5 & 7.5 & 6.4 & 5.5 & 4.3 & 3.1 & 1.8 & 0.96 \\
\hline$x$ & 0.429 & 0.463 & 0.481 & 0.503 & 0.509 & 0.511 & 0.518 & 0.534 & 0.561 & 0.578 \\
$y$ & 0.333 & 0.344 & 0.348 & 0.352 & 0.349 & 0.349 & 0.349 & 0.348 & 0.347 & 0.345
\end{tabular}

TABLE II

Colorimetric parameters of $0.57 \mu \mathrm{m}$ thick $\mathrm{ZnSe} / \mathrm{GaAs}(100)$ thin film (solid squares in Fig. 6). $I$ - current in nA. $U$ - voltage in $\mathrm{kV}$.

\begin{tabular}{|c|c|c|c|c|c|c|c|c|c|}
\hline \multicolumn{9}{|c|}{ Accelerating voltage $10 \mathrm{kV}$} & \\
\hline$I$ & 5.3 & 4.4 & 3.6 & 3.0 & 2.4 & 1.7 & 1.0 & 0.7 & \\
\hline$x$ & 0.359 & 0.367 & 0.379 & 0.381 & 0.396 & 0.419 & 0.441 & 0.471 & \\
\hline$y$ & 0.283 & 0.285 & 0.287 & 0.287 & 0.287 & 0.293 & 0.298 & 0.312 & \\
\hline \multicolumn{10}{|c|}{ Varying accelerating voltage } \\
\hline$U$ & 7 & 8 & 9 & 10 & 12 & 14 & 16 & 18 & 20 \\
\hline$I$ & 3.03 & 2.65 & 2.36 & 2.12 & 1.77 & 1.42 & 1.33 & 1.18 & 1.06 \\
\hline$x$ & 0.367 & 0.377 & 0.411 & 0.414 & 0.442 & 0.511 & 0.506 & 0.554 & 0.570 \\
\hline$y$ & 0.223 & 0.242 & 0.281 & 0.297 & 0.332 & 0.381 & 0.379 & 0.388 & 0.386 \\
\hline
\end{tabular}

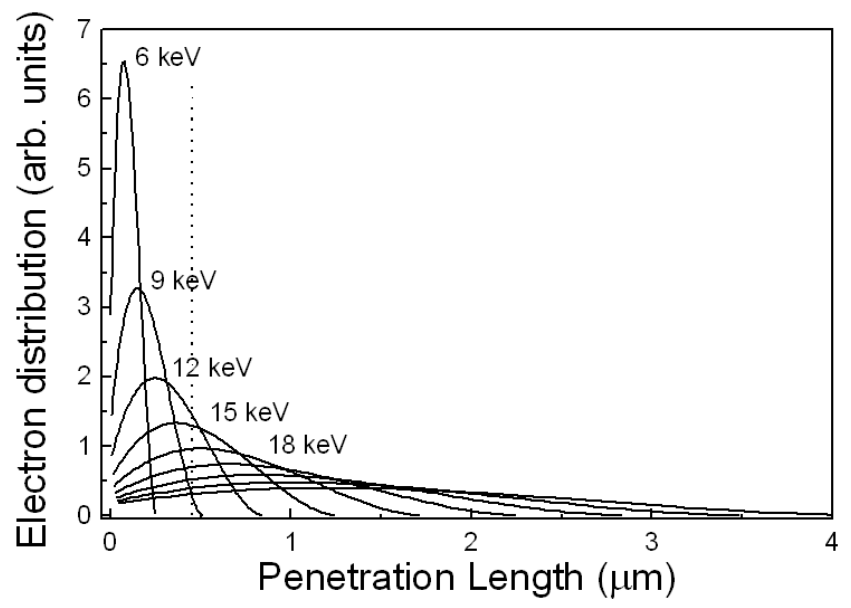

Fig. 4. The penetration range of primary electrons in ZnSe films as a function of accelerating voltage. 


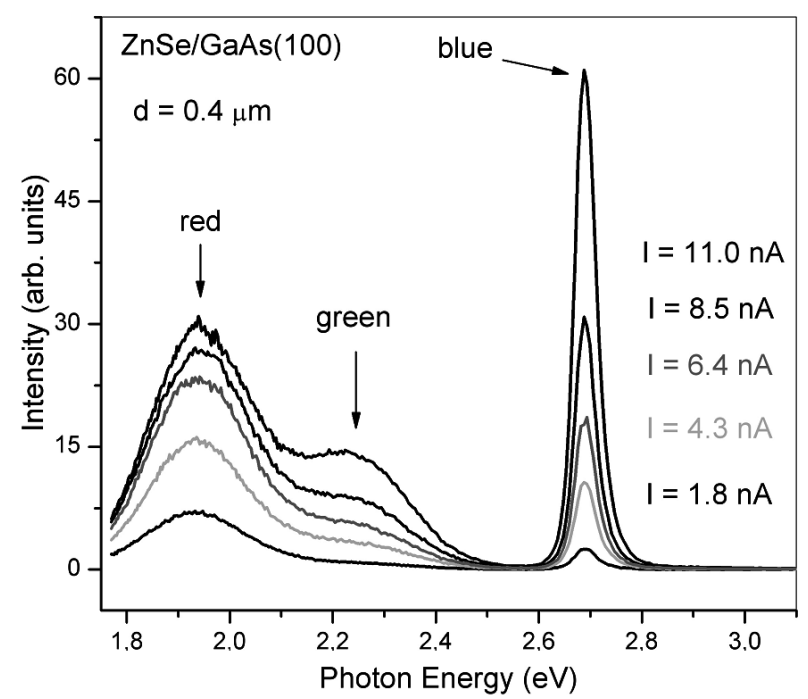

Fig. 5. CL spectra taken at accelerating voltage of $20 \mathrm{kV}$ and different current densities $(I=1.8 \div 11 \mathrm{nA})$. The contribution of "green" emission band increases with current density.

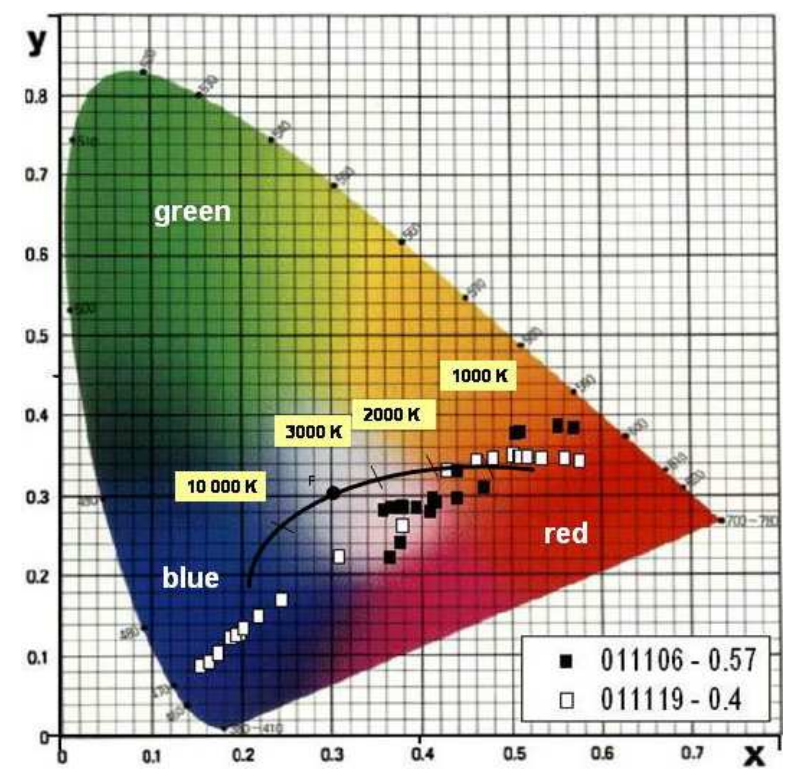

Fig. 6. CIE chromaticity diagram showing the chromaticity coordinates of investigated $\mathrm{ZnSe} / \mathrm{GaAs}(100)$ films in a function of excitation parameters (accelerating voltage and current density). Open squares correspond to the $0.4 \mu \mathrm{m}$-thick layer, whereas black squares correspond to the $0.57 \mu$ m-thick layer. Black solid line shows chromaticity coordinates of the black body radiator. 
and $y$ presented in Tables I and II are coordinates at CIE chromaticity diagram. They are also shown graphically on a chromaticity diagram (Fig. 6). Analysis of data presented in Tables I and II together with Fig. 6 lead to the conclusion that the accelerating voltage in the range of $10-20 \mathrm{kV}$ and current density between 1-20 nA applied to a sample with a thickness of about $0.5 \mu \mathrm{m}$ assure a full range of required color temperature.

\section{Conclusions}

In conclusion, optically excited thin ZnSe films deposited on GaAs substrate by atomic layer epitaxy method give bright white emission. We have shown that colorimetric parameters of the emission spectra might be adjusted by changing accelerating voltage and/or current density and required color temperature might be easily achieved. These make $\mathrm{ZnSe} / \mathrm{GaAs}$ films attractive candidates for some optoelectronic applications like backlighting of liquid crystal displays (LCDs).

\section{Acknowledgments}

The work was supported by the State Committee for Scientific Research grant no. 1 P03B 01529 of the Ministry of Science and Higher Education (Poland) granted for the years 2005-2008. The F-120 ALE reactor of Microchemistry was bought using SEZAM grant of Foundation for Polish Science.

\section{References}

[1] S. Nakamura, Proc. SPIE 3002, 26 (1997).

[2] P. Schlotter, R. Schmidt, J. Schneider, Appl. Phys. A 64, 417 (1997).

[3] K. Sato, M. Hanafusa, A. Noda, A. Arakawa, T. Asahi, M. Uchida, O. Oda, IEICE Trans. Electron. R83-C, 579 (2000).

[4] Y.A. Ono, in: Encyclopedia of Applied Physics, Eds. G.L. Trigg, R.G. Lerner, Vol. 5, VCH Publishers Inc., Weinheim 1992, p. 1.

[5] M. Godlewski, M. Leskelä, CRC Crit. Rev. Solid State Mater. Sci. 19, 199 (1994).

[6] H. Wenisch, M. Fehrer, M. Klude, K. Ohkawa, D. Hommel, J. Gryst. Growth 214/215, 1075 (2000).

[7] M.C. Tamargo, W. Lin, S.P. Guo, Y. Guo, Y. Luo, Y.C. Chen, J. Crys. Growth 214/215, 1058 (2000).

[8] M. Godlewski, E. Guziewicz, K. Kopalko, E. Lusakowska, E. Dynowska, M.M. Godlewski, E.M. Goldys, M.R. Phillips, J. Lumin. 102-103, 455 (2003).

[9] E. Guziewicz, M. Godlewski, K. Kopalko, E. Łusakowska, E. Dynowska, M. Guziewicz, M.M. Godlewski, M. Phillips, Thin Solid Films 446, 172 (2004).

[10] M. Godlewski, M. Skrobot, E. Guziewicz, M.R. Phillips, to be published in J. Lumin.

[11] K. Kopalko, M. Godlewski, E. Guziewicz, E. Łusakowska, Lusakowska, Paszkowicz, J. Domagała, E. Dynowska, A. Szczerbakow, A. Wójcik, M.R. Phillips, Vacuum 74, 269 (2004). 
[12] G. Hitier, J. Phys. 41, 443 (1980).

[13] M. Godlewski, W.E. Lamb, B.C. Cavenett, J. Lumin. 24/25, 173 (1981).

[14] M. Godlewski, E. Guziewicz, V.Yu. Ivanov, in: Handbook of Electroluminscence Materials, Ed. D.R. Vij, IOP Publishing, Philadelphia 2004, ch. 3, p. 124. 